\title{
Research on Intelligent Supervision Method and System Developing of Soil Remediation Project Based on 3D Mobile GIS
}

\author{
Bin Xie ${ }^{1}$, Xiangwei Zhao ${ }^{1, *}$, Jun Yang ${ }^{2}$, Qingzhong Wang ${ }^{1}$, and Shun Pan ${ }^{1}$ \\ ${ }^{1}$ Key Laboratory of Geomatics and Digital Technology of Shandong Province, Shandong University of Science and Technology, \\ Qingdao 266590, China \\ ${ }^{2}$ Institute of Geographic Science and Natural Resources Research, Chinese Academy of Sciences, Beijing 100864, China
}

\begin{abstract}
At present, some problems such as inconvenient manual supervision, lack of real-time online supervision and poor online interaction during the implementation of soil remediation project. In order to solve the problems, the supervision method and system development of soil remediation project are studied based on sensing online and 3D mobile GIS technologies. The remediation environment are monitored with fixed sensors online and the heavy metal content are sampled with the mobile sensors. The status of soil remediation site is supervised in real time with video online. All the real-time sensing data are integrated and stored in the comprehensive database. The information of soil pollution and remediation environment is visualized with maps and charts in 3D geographic scenes in the system. In addition, the pollution degree evaluation and remediation effect analysis functions are implemented in system. It is proved with the project practice that the method and system are convenient for managers to monitor the environment on-site and supervise the status of soil remediation site in real time. The informationization and intelligence level of soil remediation project can be effectively improved.
\end{abstract}

\section{Introduction}

In recent years, with the increasing efforts of environmental control and the effective implementation of various measures, the momentum of pollution has been curbed in China. However, pollution problems such as heavy metal pollution in soil are difficult to control and make a serious impact on China's environmental governance. It not only threatens people's health, but also causes social instability. In order to solve such problems, soil remediation experiments have been carried out in some seriously polluted areas according to the current situation of soil pollution in China[1-5]. The farmland remediation project has a large area, complex conditions and long cycle. Therefore, it is great significant to research intelligent supervision method and build soil remediation monitoring system for the managers to master the soil status and the remediation effect.

On the basis of the agricultural network system, the United States began to industrialize the application of monitoring technologies in the field of precision agriculture, fertilizer spraying and other fields. Japan mainly focuses on portable intelligent agriculture, while the Netherlands has built a greenhouse agriculture efficient production system [6-7]. South Korea and Israel set up the technical service system of agricultural Internet of things to promote the application of agricultural Internet of things supervision technology. The agricultural Internet of things develops rapidly in China and has some applications in environmental monitoring[8-14].

According to the above research, the research results have important guiding significance for the construction of soil remediation supervision system. However, the construction of soil remediation project supervision system in China is still in the initial stage. The existing soil remediation supervision system is mainly developed based on the browser/server $(\mathrm{B} / \mathrm{S})$ framework, and the response speed of such system is slow and affected by time and space. In addition, the optimization and integration of virtual reality technology, Internet of things technology, multi-sensor technology and 3D mobile GIS technology are rarely reported. With the development of mobile Internet technology and the improvement of the performance of mobile hardware devices (high-definition display screens, graphics processing units and solid-state drives), the development of mobile monitoring systems has become possible[15-16]. By integrating the real-time monitoring information of the Internet of things with the mobile virtual reality scene, managers can obtain monitoring information in real time. Therefore, it is necessary to design a soil remediation monitoring system based on 3D mobile GIS. Through this system, the functions of quick query of soil environment data, automatic warning of outliers, evaluation of remediation effect and 3D interaction can be realized on the mobile terminal.

\footnotetext{
* Corresponding author: zhaoxwchina@sdust.edu.cn
} 


\section{Supervisory system design}

\subsection{System framework design}

Managers need to personally guide the implementation of soil remediation. However, due to the large area and long regulation process, it is impossible for technicians and managers to conduct comprehensive on-site supervision. Therefore, it is necessary to establish a mobile terminal 3D scene to improve the user's sense of communication. In addition, the occurrence of emergency is the reason that the alarm information cannot be obtained by the staff, and the correct guidance and treatment cannot be issued. Therefore, the real-time transmission and expression of data are the basis of the supervisory system.

To solve the above problems, the system adopts the mobile terminal/server framework. The system framework includes user layer, presentation layer, business logic layer and data layer(Figure 1).

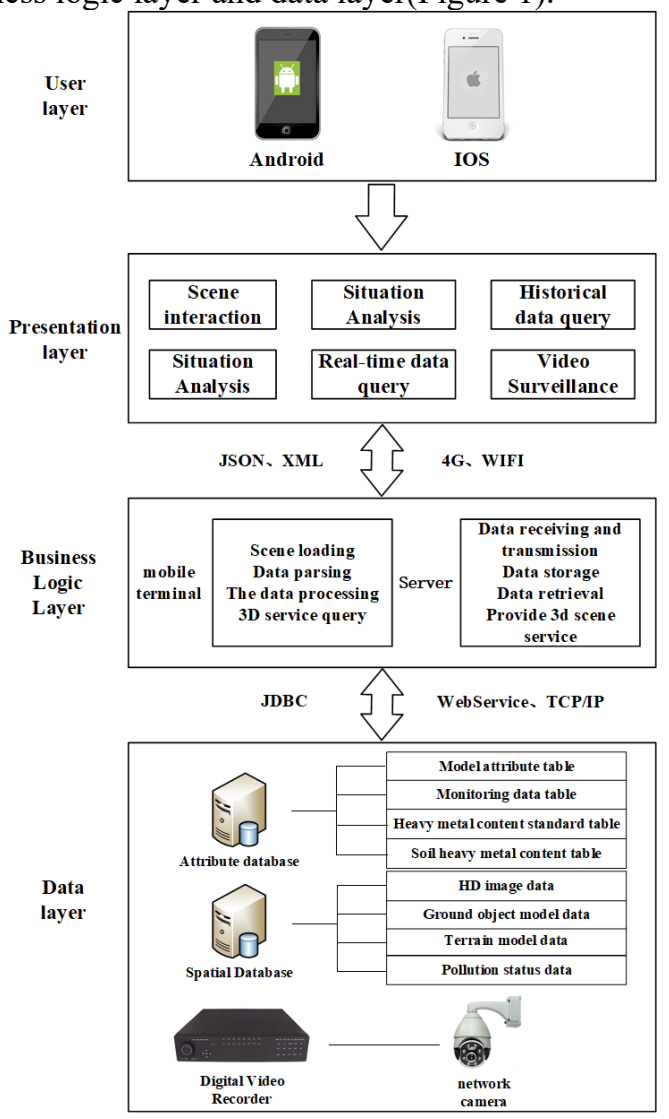

Fig.1. Framework of the system

The user layer is carried by smart phones. It stores the $3 \mathrm{D}$ virtual geographic scene cache containing terrain, ground objects and other elements on the mobile client, which improves the scene loading speed. Sensor data collection, field video and other perceptual information can be integrated with 3D terrain, remote sensing image, thematic map of pollution status and other spatial data on the mobile phone, so as to realize the analysis and query of spatial data.

The presentation layer is the basic function implemented by the mobile terminal. It includes visualization of perceptual data, analysis of remediation status, analysis of remediation changes and 3D interaction.

Business logic layer carries out business processing, which is mainly completed by mobile terminal and server terminal. The mobile terminal is responsible for providing an interactive visual interface for the user layer, which implements such functions through specific technologies and services. The main functions of the server include data reception and transmission, data access, data retrieval and providing 3D scene services.

The data layer includes property database, spatial database and video data stored by digital video recorder. It mainly provides data support for the business layer. Among them, Oracle database is the adopted property database. Map services and 3D services are published through the SuperMap iServer 9D platform, which provides mobile network spatial data services. Video data is collected by network camera, which is transmitted to digital video recorder through wired network, and it is processed and stored by digital video recorder.

\subsection{D scene construction and release}

It is difficult to construct 3D scene because of its wide geographical range, high terrain accuracy, large amount of terrain data and large number of ground object models. In addition, the mobile system requires the smooth transmission of network data, so it is very important to choose a high-performance development platform. SuperMap platform supports the storage and fast access of massive 3D spatial data. When loading the ground object model, it can flexibly switch different levels of ground object texture by visual distance, and can publish 3D data on the network and transmit it in a stream mode. Therefore, SuperMap platform is used for secondary development in this study. This platform supports the storage of 3D data cache on the mobile terminal, which speeds up the loading speed of 3D scenes and gives users a good interactive feeling. The process of $3 \mathrm{D}$ scene construction and release is shown in Figure 2.

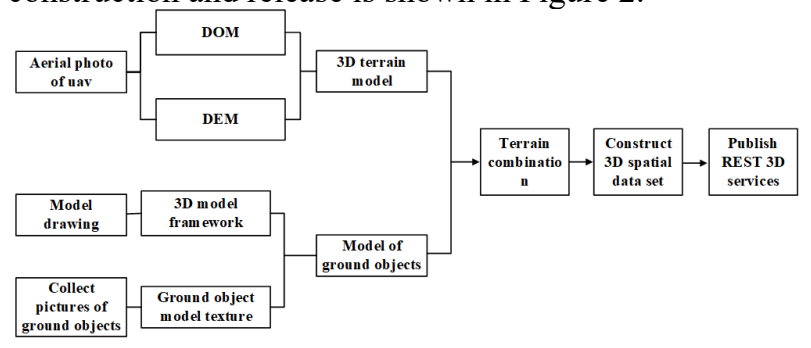

Fig.2. Flow chart of 3D scene construction and release

1) Terrain model

DEM (Digital Elevation Model) and DOM (Digital Orthophoto Map) with high resolution in the soil remediation area were acquired by uav aerial photography. DEM and DOM data are processed by projection transformation, geometric correction and cropping[17-18], and then the visual effect of DOM is improved by means of color enhancement and rendering. The processed data are imported into SuperMap iDeskTop, where DEM is the topographic map layer and DOM is the raster image layer. 
Then the two are superimposed to construct a high precision 3D terrain model with spatial reference.

2) Ground object model

Simple models of buildings can be built in SketchUp, and sophisticated models of multi-sensor observatories and webcams can be built in 3D Max. The photos of various buildings and monitoring equipment collected in the field are spliced together, and the color and resolution of the pictures are adjusted. Then the processed pictures are pasted on the 3D model as the model texture. After the placement of the ground object model, the file format of the ground object model can be converted into OSGB (Open Scene Gragh Binary) format which can display hierarchical details through the conversion tool of SuperMap iDeskTop[19]. The model in this format can automatically adjust the texture precision according to the visual distance to improve the loading speed of the model in the 3D Scene.

\section{3) Release of 3D scene}

According to the coordinate data collected by GPS, the ground object models, such as buildings and observation stations, were integrated into corresponding positions of 3D terrain. Finally, the whole scene was published on the GIS server as a REST (Representational State Transfer)3D service. The published scene can be loaded into a browser for viewing through the REST service address, or it can be loaded into the SuperMap iDeskTop for editing. After the release of $3 \mathrm{D}$ scene data set, the mobile 3D virtual geographical scene of soil restoration area was built based on SuperMap iMobile SDK and 3DGIS technology.

\subsection{Data integration}

\subsubsection{Data acquisition}

In the construction of mobile terminal 3D scene, various environmental sensing data and video field data of different plots need to be integrated with 3D geographical scene. Therefore, real-time and continuous acquisition of various sensing data is an important basis for realizing intelligent supervision of soil remediation projects. Realtime data acquisition is to use the Internet of things sensor technology to collect real-time environmental data and field video data in the soil remediation area, and realize real-time transmission, storage and remote access of the data.

According to the actual requirements of soil remediation project, the real-time environment sensing system is divided into sensing layer, network transmission layer and application layer. The environment - aware system architecture is shown in Figure 3.

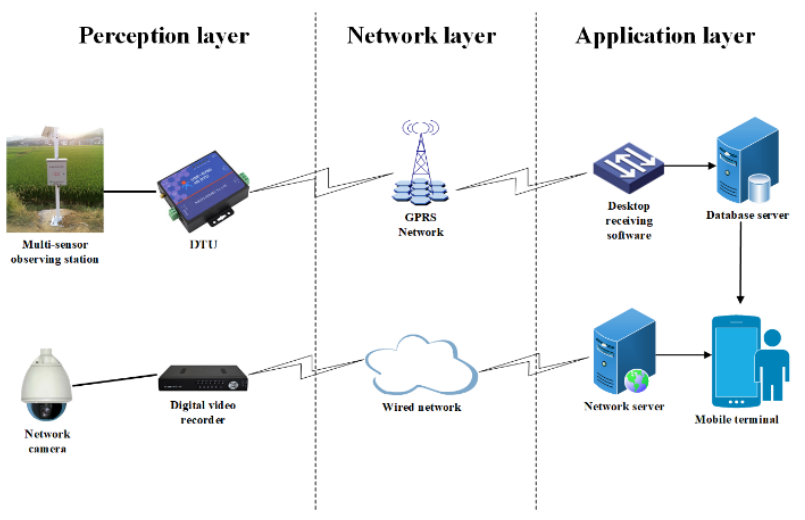

Fig.3. Environment - Aware System Architecture

1) The perception layer is used to acquire multi-type sensor data and monitor video data in different areas. Monitoring equipment of the observation station includes multi-type perceptron, DTU (Data Transfer unit) module, power supply and protection box, etc. Real-time data collected by various types of sensors are applied in the location specified in the remediation area, and transmitted by GPRS(General Packet Radio Service, GPRS) module built in DTU. Video monitoring equipment is composed of network camera, network switch and digital video recorder.

2) The network transmission layer is responsible for uploading the sensing information to the application layer, and issuing the relevant commands of the control sensor of the application layer to the perception layer. Considering the small amount of monitoring data collected by multiple types of sensors and the relatively weak network infrastructure in farmland areas, it is suitable to use stable and reliable GPRS wireless network for data transmission. The sensor perceptron is connected to the GPRS module, which transmits the data acquired by the perceptron to the data receiving software of the application layer based on TCP/IP protocol. Network camera has a high frequency of data acquisition and a large amount of data, so video data is transmitted by wire transmission. The network camera is connected to the network switch via twisted pair, which is connected to the digital video recorder storing video data.

3) The application layer provides core services for environmental monitoring of soil remediation project, consisting of a database server, a web server and a mobile terminal. To facilitate the reception of environment-aware data, sensor desktop reception software was developed in the database server (Figure 4). The software connects DTU with the server through computer serial port, and realizes the parsing and storage of request instructions. Web server developed a webservice program, it is the bridge that mobile terminal can request data from the database server. The mobile terminal, as the client to display the 3D virtual geographic scene of the network, can view all kinds of monitoring data in real time and remotely control the monitoring equipment. 


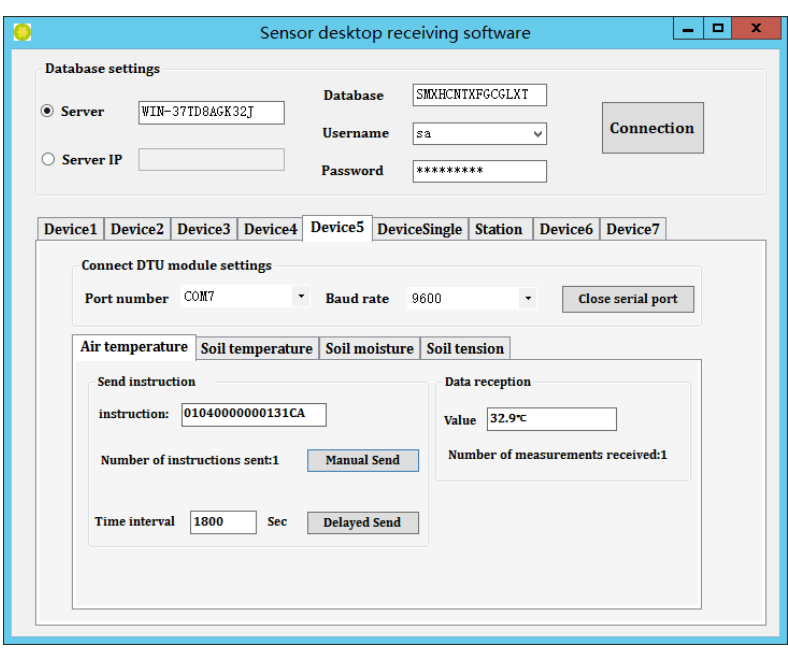

Fig.4. Sensor desktop receiving software

\subsubsection{Spatial Data Integration}

The integration of multi-sensor data and video data with $3 \mathrm{D}$ scene is mainly realized by unique identifier. Specific implementation methods: Firstly, the device number or camera number information stored in the model is obtained by clicking the observation station model or camera model in the 3D scene, and the acquired serial number information is saved as a unique identifier. Secondly, pass the unique identifier to the corresponding active component (such as the corresponding active component Camera-Activity of Android system and the corresponding active object Camera-View-Controller of IOS system). Finally, the system uses the fields such as identifier, date, sensor type and so on as the filter conditions of the query database to display the results of the query in the new interface.

The spatial database engine SuperMap SDX+ is used to realize the combination of heavy metal sampling data and $3 \mathrm{D}$ scene. This engine uses $\mathrm{r}$-tree spatial index to improve data access speed, and r-tree index has high spatial retrieval efficiency and precision, which is conducive to improving response speed. Implementation method: Firstly, the collected results of heavy metal pollution in various regions are recorded into the corresponding property sheet of the thematic map of the pollution status in the remediation area. Then the thematic map is superimposed on the scene and published as a three-dimensional service. Finally, SuperMap SDX+ imports the data from the the thematic map of the pollution status into the property database and automatically establishes a spatial index for it. After the establishment of spatial index, the data in the thematic map of soil pollution will be associated with the property database, so as to realize the integration of the data of soil status and the three-dimensional geographical scene of mobile terminal.

\section{Implementation of 3D supervision system}

\subsection{Function Implementation of supervisory system}

\subsubsection{Heavy metal pollution evaluation}

1) Pollution status evaluation

According to national standards, the evaluation method of metal pollution is divided into five levels: clean, trace pollution, light pollution, moderate pollution and heavy pollution[20]. The single-factor index method is commonly used for evaluation. The single-factor index is calculated as following equations:

$$
\begin{aligned}
& P_{\mathrm{i}}=C_{\mathrm{i}} / S, \quad \mathrm{i}=1,2, \ldots, \mathrm{n} \\
& P=\max \left(P_{\mathrm{i}}\right)
\end{aligned}
$$

where $P_{\mathrm{i}}$ denotes the single factor index, $C_{\mathrm{i}}$ denotes the measured concentration of a heavy metal, $S$ denotes the heavy metal standard, i denotes the number of pollutants. The maximum value of single factor index $P_{\mathrm{i}}$ of each pollutant is the single factor index $P$ of this point. Standards for evaluating pollution degree according to $P$ value are shown in Table 1 [21].

Table 1. Pollution Degree Evaluation Criteria

\begin{tabular}{|c|c|c|}
\hline $\begin{array}{c}\text { Super standard } \\
\text { multiple }\end{array}$ & $\begin{array}{c}\text { Degree of soil } \\
\text { pollution }\end{array}$ & $\begin{array}{c}\text { Soil environmental } \\
\text { quality category }\end{array}$ \\
\cline { 1 - 2 } $\mathrm{P} \leq 1.0$ & clean & $\begin{array}{c}\text { Priority protection } \\
\text { class }\end{array}$ \\
\hline $1.0<\mathrm{P} \leq 2.0$ & Trace pollution & \multicolumn{1}{|c}{} \\
\hline $2.0<\mathrm{P} \leq 3.0$ & Light pollution & Safe utilization class \\
\hline $3.0<\mathrm{P} \leq 5.0$ & Moderate pollution & \\
\hline $\mathrm{P}>5.0$ & Severe pollution & Strict management \\
\hline
\end{tabular}

The pollution status evaluation is implemented as the following steps. First, an array of objects is created to store the evaluation results of the sample points. The array of objects is traversed to extract the heavy metal content data from each sampling point. Then the data are used to calculate the "excess multiple" and "pollution degree" of each heavy metal pollutant, and the "farmland environmental quality category" at the sampling site. The evaluation results are assigned to objects in the object array in the form of object properties. After traversal, all the sampling points are evaluated, and the results of the evaluation are stored in an array of objects. The evaluation results were presented in five different colors according to the degree of soil pollution. By clicking the analysis and evaluation button, render the scene block, and check the pollution degree of the block according to the legend. Then click the block in the scene to check the soil pollution status of the block. The results of pollution status are shown in Figure 5(a).

2) Remediation effect evaluation

This function aims to analyze the changes of monitoring data in each block within a certain period of time and generate a soil remediation change map (Figure 5(b)). If the heavy metal concentration of a certain block decreases significantly within a certain time, the block will be rendered with green color. Instead, the block is 
rendered in red. If the change is not obvious, render the block in yellow. When comparison button is clicked, the system will calculate the earliest monitoring data and the latest monitoring data to generate a pollution change layer. The layer is superimposed on top of the terrain model. The results show that: by comparing the data of 2012 and 2018 , it can be seen that after several years of treatment, the heavy metal pollution index of the remediation area decreased significantly, and the soil remediation effect was obvious.

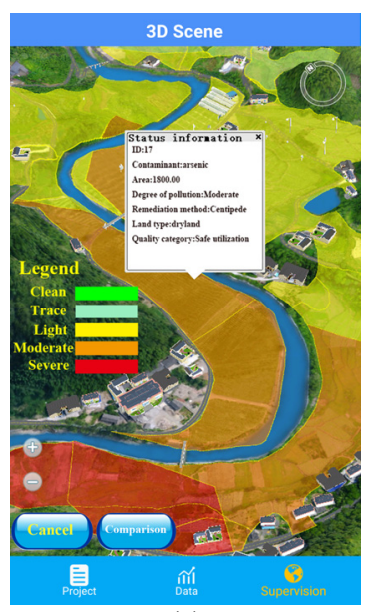

(a)

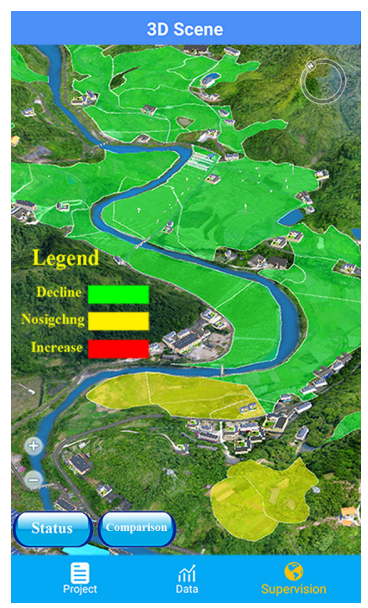

(b)
Fig.5. Remediation effect of heavy metal

\subsubsection{Real-time perceptive data visualization}

1) Video monitoring online

Through this function, users can access video information of cameras in different areas, and can make basic control of the camera, such as zoom, lens movement and so on. As is shown in Figure 6. Figure (a) represents the distribution of cameras in the three-dimensional scene, and Figure (b) shows monitoring video and its console when the user clicks on the model.

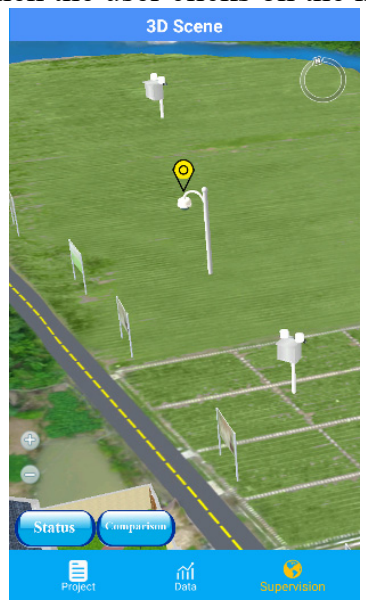

(a)

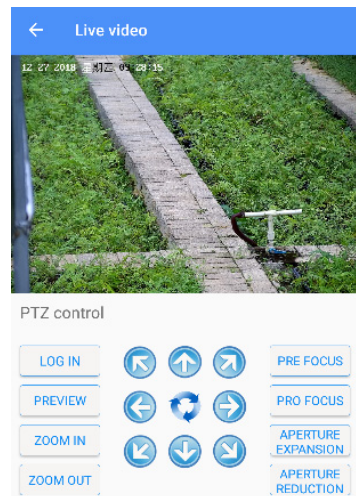

(b)
Fig.6. Real-time monitoring video in 3D scene

2) Real-time monitoring of environmental data

Users can view historical and real-time data from the observation station based on sensor type, date range and time interval, which is presented in the form of dynamic charts. The implementation process is descripted as the folloing. The mobile terminal retrieves database data through WebService, and then returns the data through JSON and XML to the front mobile terminal, and the mobile terminal displays the results through Echart in the $3 \mathrm{D}$ scene through data parsing and processing[22]. Taking air temperature monitoring data query as an example, the effect diagram is shown in Figure 7. Figure (a) represents the distribution of observation station model in the threedimensional scene, Figure (b) shows the real-time air temperature data of the observation station.

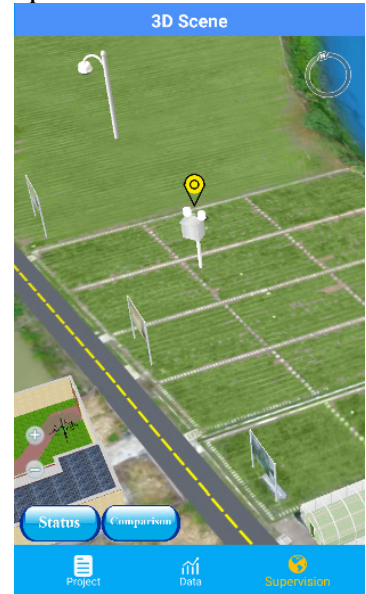

(a)

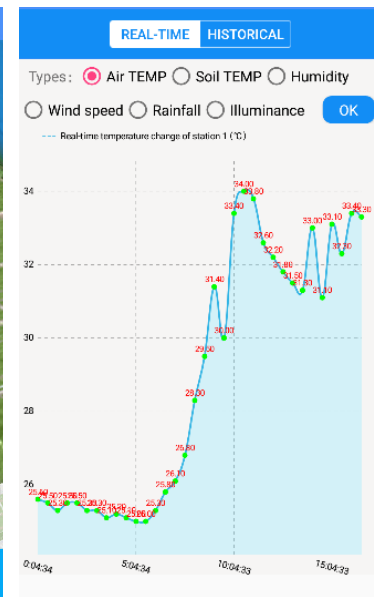

(b)
Fig.7. Environmental monitoring data querying

3) Monitoring and warning system

The warning system is based on real-time monitoring data. The sensor in the $3 \mathrm{~d}$ geographic scene is correlated with the latest monitoring value of the sensor in the database. When the monitoring value of the sensor is lower or higher than the alert value, positioning markers will appear in the position of the sensor model, so that managers can take corresponding measures in time. Warning information is transmitted to the user in the form of sound and light alarm and SMS (Short Message Service). The process of environmental monitoring and warning is shown in Figure 8. 


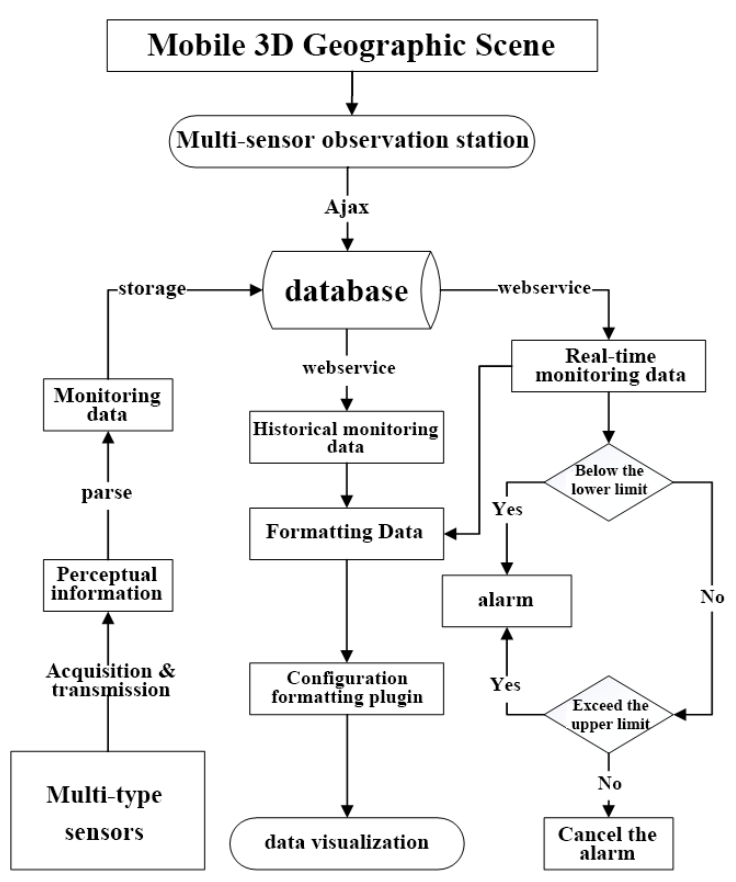

Fig.8. Monitoring and Warning Process

\section{Conclusions}

Due to the lack of application of 3D mobile GIS and sensor technology in the field of soil remediation supervision, it is not convenient to monitor the real-time environment and pollution status of soil remediation project effectively and intuitively. Mobile 3D GIS technology, sensor technology, network communication technology, 3D virtual reality and other related technologies are effectively integrated to study the remote intelligent supervision method of soil remediation project in this article. Combined with the business requirements of soil remediation, the functions of $3 d$ visualization of perceived data, real-time monitoring and early warning of soil environment, evaluation of pollution status and evaluation of remediation effect are realized. The problems existing in the current soil remediation supervision informatization have been effectively solved.

\section{References}

1. G.T. Zhuang, Current situation of national soil pollution and strategies on prevention and control, Bulletin of C-hinese Academy of Sciences, 30, 477483(2015)

2. J. Zhou, H.B. Cui, J.N. Liang, Remediation technologies and current problems of heavy metal contaminated sites with "demonstration project of soil remediation on the periphery of Guixi smelter" as example, Soils, 47 , 283-288(2015)

3. T.B. Chen, J. Yang, M.Lei, Soil remediation project of arsenic pollution farmland in Shimen county, Hunan province, World Environment, 4 , 57-58(2016)

4. X.G Meng, C. Xu, Y.Q. Yang, Soil remediation project instances of some chromium residue contaminated sites in Yunnan, Chinese Journal of Environmental Project, 11 ,6547-6553(2017)

5. D.M. Li, Y.H. Gong, Daye city, Hubei province national farmland soil restoration demonstration project, World Environment, 4, 54-56(2016)

6. J. Li, M.R. Guo, L.L. Gao, Application and innovation strategy of agricultural Internet of Things, Transactions of the Chinese Society of Agricultural Project, 31 ,200-209(2015)

7. A. Kaloxylos, R. Eigenmann, F. Teye, Farm management systems and the Future Internet era, Computers and electronics in agriculture, 89, 130144(2012)

8. X.Y. Liu, Thoughts about developing regional agriculture economic in guizhou--learning from netherlands and israels experiences of developing agriculture, Chinese Journal of Agricultural Resources and Regional Planning, 3,75-80 (2009)

9. X.M. Zuo, W.1. Gao, G.H. Zhang, Design of Environmental Parameters Monitoring System for Watermelon Seedlings Based on Wireless Sensor Networks, Applied Mathematics \& Information Sciences, 5, 243-250(2011)

10. S.P. Zhu, M.H. Deng, J. Feng, Mobile InternetTechnology Based on Farmland Ecological Environment Research of Remote Monitoring System, Journal of Agricultural Mechanization Research, 3811,224-228(2016)

11. L.F. Lin, R.S. Wang, P.H. Yu. GIS based visual monitoring system of farmland microclimate environment, Transactions of The Chinese Society of Agricultural Machinery, 46, 254-260(2015)

12. J.S. Liao, Design of agricultural greenhouse environment monitoring system based on internet of things, Transactions of the Chinese Society of Agricultural Engineering, 3211, 233-243(2016)

13. Z.G. Sun, Y.S. Wang, L. Zhang, W. Guo, Design and realization of intelligent service system for monitoring and warning of meteorological disasters in facility agriculture in North China, Transactions of the Chinese Society of Agricultural Engineering, 34, 149-156(2018)

14. J. Lu, G.F. Chen, Y. Meng, Simulation of farmland environmental monitoring based 3D GIS and system implementation, Journal of Agricultural Science and Technology, 19, 50-55(2017)

15. J.Y. Wu, W.J. Li, J.P. Huang, Key techniques for Mobile Internet: a survey, Scientia Sinica(Informationis), 4501,45-69(2015)

16. J.Y. Jiao, T. Li, H.M. Du, State of the Art and Development of Mobile Graphics Processing Unit. Journal of Computer-Aided Design \& Computer Graphics, 27, 1005-1016(2015)

17. L.J. Han, S.W. Li, The Principle and Application Elementary Analysis of Projection Transformation, Science\&Technology Vision, 26,171-174(2015) 
18. D.M. Xiang, SPOT image geometric precise correction with DEM auxiliary, China University Of Geosciences(2009)

19. Y.M. Guo, Z.J. Cui, Y.H. Lu, A Survey: Technologies of LOD in Virtual Environment Generation, Computer Simulation, 12, 180-184(2005)

20. J. Li, Y. Chang, S.Y. Pan,Evaluation Methods of Soil Heavy Metal Pollution, Journal of Agricultural Catastrophology, 2, 50-52+72(2012)

21. S.X. Fan, Z.T. Gan, M.J. Li, Progress of Assessment Methods of Heavy Metal Pollution in Soil. Chinese Agricultural Science Bulletin, 26, 310-315(2010)

22. N.Jia, Application of Echarts in Mobile Data Communication, MobileCommunications, 40, 50-53 (2016) 